\title{
Laparoscopic Toupet Fundoplication After Roux-en-Y Gastric Bypass in Patient with Severe GERD
}

\author{
Peter Vorwald ${ }^{1} \cdot$ Rafael Mauricio Restrepo Núñez ${ }^{1}$ (D) $\cdot$ Gabriel Salcedo Cabañas ${ }^{1} \cdot$ Maria Posada González $^{1}$
}

Published online: 6 February 2019

(C) Springer Science+Business Media, LLC, part of Springer Nature 2019

\begin{abstract}
Background Up to $22 \%$ of the patients, after laparoscopic Roux-en-Y gastric bypass (LRYGB) can complain about gastroesophageal reflux disease (GERD) symptoms, there is little evidence about the most effective treatment of this situation.

Materials and Method This video shows a laparoscopic reintervention after a LRYGB in a 42-year-old woman presenting with untreatable GERD. GERD was treated with maximum doses of PPIs with no effect on the symptoms; the patient had a 24-h pH and impedance manometry that showed an acid and non-acid reflux, and for that reason, we decided to perform a laparoscopic fundoplication. First, very dense adhesions of the greater omentum and the stomach to the parietal peritoneum and the left lobe of the liver are dissected. The gastric remnant is dissected from the pouch, and as the adhesions are very hard, we use a linear stapler device to definitely separate remnant from the pouch, then, short gastric vessels are taken down, to allow mobilization to the medial side of the patient, being careful to preserve the dorsal gastric artery branch of the splenic artery. A Toupet fundoplication is performed with the gastric remnant, and a crural repair is performed with Ethibond stitches reinforced with a polypropylene mesh. Results After 1 year from the surgery, the patient is asymptomatic, with a normal $\mathrm{pH}$ metry and barium swallow.

Conclusions The laparoscopic fundoplication [1] after a LRYGB is one feasible surgical option intervention, but it must be performed with experienced hands and in a hospital with bariatric/esophagogastric surgery unit, in those patients with severe GERD after LRYGB and with no response to medical treatment.
\end{abstract}

Keywords GERD $\cdot$ Obesity $\cdot$ Toupet $\cdot$ LRYBP $\cdot$ Surgery

\section{Compliance with Ethical Standards}

All procedures performed in studies involving human participants were in accordance with the ethical standards of the institutional and/or national research committee and with the 1964 Helsinki declaration and its later amendments or comparable ethical standards.

Conflict of Interest The authors declare that they have no conflict of interest.

Electronic supplementary material The online version of this article (https://doi.org/10.1007/s11695-018-03691-6) contains supplementary material, which is available to authorized users.

Rafael Mauricio Restrepo Núñez

rafaelrestreponunez@gmail.com

1 Surgeon Esophagogastric Unit, Hospital Fundación Jiménez Díaz, Madrid, Spain
Publisher's Note Springer Nature remains neutral with regard to jurisdictional claims in published maps and institutional affiliations.

\section{References}

1. Kawahara NT, Alster C, Maluf-Filho F, et al. Modified Nissen fundoplication: laparoscopic antireflux surgery after Roux-en-Y gastric bypass for obesity. Clinics (Sao Paulo). 2012;67:531-3. 